\title{
Prognostic role of neutrophil-to-lymphocyte ratio in diffuse large B cell lymphoma patients: an updated dose-response meta-analysis
}

\author{
Shidai $\mathrm{Mu}^{1+}$, Lisha $\mathrm{Ai}^{1 \dagger}$, Fengjuan Fan ${ }^{1}$, You Qin ${ }^{2}$, Chunyan Sun ${ }^{1^{*}}$ and Yu Hu${ }^{1 *}$
}

\begin{abstract}
Background: The neutrophil-to-lymphocyte ratio (NLR), a biomarker for systematic inflammation, has been recently identified as a prognostic factor for various types of both solid and hematologic malignancies. Here we conducted an updated dose-response meta-analysis to investigate whether NLR can be served as a prognostic biomarker in diffuse large B cell lymphoma (DLBCL).

Methods: We systematically searched PubMed, Embase, ISI Web of Science and CNKI for relevant studies. Odds ratios or hazards ratios (HRs) with corresponding 95\% confidence intervals (Cls) were pooled to estimate the association between NLR and clinicopathological parameters or survival of cancer patients.

Results: Eleven trials with 2515 DLBCL patients were included in the meta-analysis. The results revealed that elevated pretreatment NLR was significantly associated with elder age, advanced Ann Arbor stage, higher incidence rate of B symptoms and bone marrow involvement, and higher lactate dehydrogenase level, etc. Moreover, increased NLR also predicted poorer overall survival (HR 1.826, 95\% Cl 1.238-2.692) and progression-free survival/event-free survival (PFS/EFS) (HR 1.591, 95\% Cl 1.124-2.252). And two-stage dose-response meta-analysis revealed non-linear association between increased NLR and risk of mortality in DLBCL patients.
\end{abstract}

Conclusion: DLBCL patients with higher NLR are more likely to have poorer prognosis than those with lower NLR.

Keywords: Neutrophil-to-lymphocyte ratio, Diffuse large B cell lymphoma, Prognosis, Meta-analysis

\section{Background}

Diffuse large B cell lymphoma (DLBCL) is the most commonly occurring form of lymphoma, which occupies approximately $30-40 \%$ of preliminary diagnosed non-Hodgkin's lymphomas (NHL). Despite the survival outcome of DLBCL patients has significantly improved [1] by the addition of rituximab immunotherapy to standard cyclophosphamide, doxorubicin, vincristine, and prednisone (CHOP) chemotherapy (R-CHOP) [2],

\footnotetext{
*Correspondence: suncy0618@163.com; dr_huyu@126.com

tShidai Mu and Lisha Ai contributed equally to this work

${ }^{1}$ Institute of Hematology, Union Hospital, Tongji Medical College,

Huazhong University of Science and Technology, Wuhan 430022, China

Full list of author information is available at the end of the article
}

approximately thirty percent patients with advanced stage of DLBCL remain intractable and the disease could relapse [3]. Prognostic assessment is important for designing of individualized therapy in DLBCL on the basis of accurate estimation of outcome.

A number of prognostic factors [4] have been studied, such as gene expression profiling [5], immunohistochemistry-based detection of prognostic biomarkers [6] and early interim analysis with positron emission tomography [7] following the initiation of R-CHOP therapy. However, many of these prognostic means are costly, difficult to perform, or not easily interpreted. Therefore, another prognostic models for DLBCL, which are inexpensive, widely available, and easily interpreted, are urgently needed for clinicians. 
Recently, numerous studies have demonstrated that the ratio of different kinds of peripheral blood cells can be used to predict prognosis of lymphoma $[8,9]$, such as lymphocyte-to-monocyte ratio (LMR), neutrophilto-lymphocyte ratio (NLR) and platelet-to-lymphocyte ratio (PLR), etc. Moreover, previous studies [10-13] have showed the relationship between increased pretreatment NLR and poor prognosis in different tumors. However, due to the divergence in the study design and sample size, direct impact of NLR level on DLBCL patients' survival and tumor's clinicopathological parameters remains inconclusive. In this study, we searched PubMed and Embase and Web of Science databases for the relevant studies and performed a meta-analysis in order to determine the association between NLR and some clinicopathological parameters, as well as the prognostic role of NLR in DLBCL.

\section{Materials and methods}

\section{Search strategy and selection of studies}

This meta-analysis was carried out in accordance with the standard guidelines for meta-analyses and systematic reviews of tumor marker prognostic studies [14, 15]. Relevant studies published before September, 2017 (date last searched), were identified through electronic searches using PubMed, Embase, and Web of Science databases. The following search terms were used: (1) "DLBCL" (e.g. "diffuse large B cell lymphoma" "B cell lymphoma" "lymphoma"); (2) "NLR" (e.g. "neutrophil to lymphocyte ratio" "neutrophil lymphocyte ratio" "neutrophil-to-lymphocyte ratio"); (3) "prognosis" (e.g. "outcome" "survival" "mortality" "recurrence"). Electronic searches were supplemented by scanning reference lists of articles identified for all relevant studies.

Studies were considered eligible if they met the following criteria: (1) patients were histopathologically diagnosed with DLBCL according to World Health Organization criteria [16]; (2) correlation of pretreatment NLR with overall survival (OS) or progression free survival (PFS) was reported; (3) Studies failing to report hazard ratio (HR) and 95\% confidence interval (CI) were included with sufficient data for estimation [17]; (4) exclusion of abstracts, letters, reviews, case reports, and multiple published reports. When there were several reports concerning the same cohort we included the high quality and most recent publication in our meta-analysis.

All initially identified studies were screened of titles and/or abstracts; then full texts were retrieved for studies that satisfied all selection criteria. Each study was independently assessed for inclusion by two reviewers $\mathrm{Mu}$ and $\mathrm{Ai}$ ) and discrepancies within the reviewing pair were resolved by the third party (Sun and $\mathrm{Hu}$ ).

\section{Data collection and extraction}

We used a predesigned data abstraction form to extract relevant information. The following details were extracted: (1) the family name of first author, year of publication, country (region) of the population studied, sample size, patient age, gender, follow-up period; (2) clinicopathological parameters including Ann Arbor stage, etc.; (3) survival data including OS and PFS/EFS; (4) cut-off value for defining "elevated NLR". OS was defined as the interval between the medical treatment and the death of patient or the last follow-up. PFS was calculated from the date of treatment until the detection of the recurrence tumor or death from any cause.

\section{Quality assessment}

A quality assessment was independently performed in each of the included studies by two reviewers (Fan and Qin) using the Newcastle-Ottawa Quality Assessment Scale (NOS) [18]. This scale uses a star system (with a maximum of nine stars) to evaluate a study in three domains: selection of participants, comparability of study groups, and the ascertainment of outcomes of interest. We judged studies that received a score of seven or more stars to be at low risk of bias, and those that scored less than seven to be at high risk of bias. This cut-off point was chosen according to the distribution of relative quality scores of all included studies. Any disagreement was resolved by the third party (Sun and $\mathrm{Hu}$ ).

\section{Statistical analysis}

HR and 95\% CI were obtained directly from each literature or from estimation according to the methods by Parmer et al. [17]. The combined rate (odds ratio, OR) and its $95 \% \mathrm{CI}$ were used to evaluate the strength of association between NLR and clinicopathological parameters.

Heterogeneity among included studies was checked by the $X^{2}$-based $Q$ test and $\mathrm{I}^{2}$ test. If there was no significant heterogeneity between studies $\left(p>0.10, \mathrm{I}^{2}<40 \%\right)$, the fixed-effect model was used. Otherwise, the randomeffects model was chosen. All statistical tests were two sided and the significance level was set at $5 \%$.

A two-stage dose-response meta-analysis was conducted to assess whether NLR was associated with higher risks of mortality from DLBCL, based on specific cut-off values, distribution of death cases and person years, and adjusted HRs with 95\% CIs. We use the generalized least square regression described by Orsini et al. [19] to calculate the specific-study linear trend and 95\% CIs for higher NLR within each study from the natural logs of adjusted HRs and 95\% CIs, and pooled HRs and 95\% CIs were obtained under the random-effects model. We approximately derived person years from follow-up duration and 
the number of participants at each NLR level. The midpoint of the higher NLR category was set at 1.2 times the lower boundary (specific cut-off value in each study). And we set the lower boundary to zero in the lower NLR category. We evaluated a potential curve linear association between NLR and risks of mortality from DLBCL, using restricted cubic splines with three knots at percentiles 10, 50, and $90 \%$ of the distribution [20].

Because characteristics of populations, ascertainment of cut-off value and adjustments for confounding factors were not consistent between studies, we further conducted a sensitivity analysis by removing one or several studies to explore possible explanations for heterogeneity and to examine the influence of various exclusion criteria on the overall risk estimate.

Assessment of publication bias was first evaluated by the Begg's funnel plot, and then performed for each of the pooled study groups using Egger's bias indicator test [21]. All analyses were carried out using STATA statistical software package version 14.0 (STATA, College Station, TX).

\section{Results}

\section{Selection and characteristics of included studies}

The flow diagram of the literature search was shown in Fig. 1. The initial search algorithm retrieved a total of 88 studies. After excluding the duplicates $(\mathrm{n}=36)$; abstracts, letters, reviews, etc. $(n=13)$; and the studies not related to research topics $(n=15)$, the remaining studies $(n=24)$ were reviewed by reading the full text. Additional studies were then excluded because they were not relevant to DLBCL $(n=8)$, not relevant to NLR $(n=3)$, not clinical research about human $(n=1)$, or repeated report about the same cohort $(\mathrm{n}=1)$. Therefore, 11 studies $[8,9$, 11, 22-29] between 2010 and 2017 with a total of 2515 DLBCL patients were enrolled in our meta-analysis.

The characteristics of the included studies were summarized in Table 1 . The publication periods of all included studies ranges from 2010 to 2016. Eight studies were from the eastern region (5 from China and 3 from Korea), and three from the western region (1 from the United States, 1 from Austria, and 1 from Croatia). Six studies enrolled $<200$ patients and five studies had $>200$ patients. 6 studies were at low risk of bias (NOS $\geq 7$ ), the rest five studies were at high risk of bias $(\mathrm{NOS}<7)$.

\section{Association between NLR and clinicopathological parameters}

Among eleven studies in our meta-analysis, eight [8, 23-29] presented a significant association between NLR and Ann Arbor staging of DLBCL patients (AA IIIIV vs. AA I-II: pooled OR 2.121, 95\% CI 1.367-3.293),

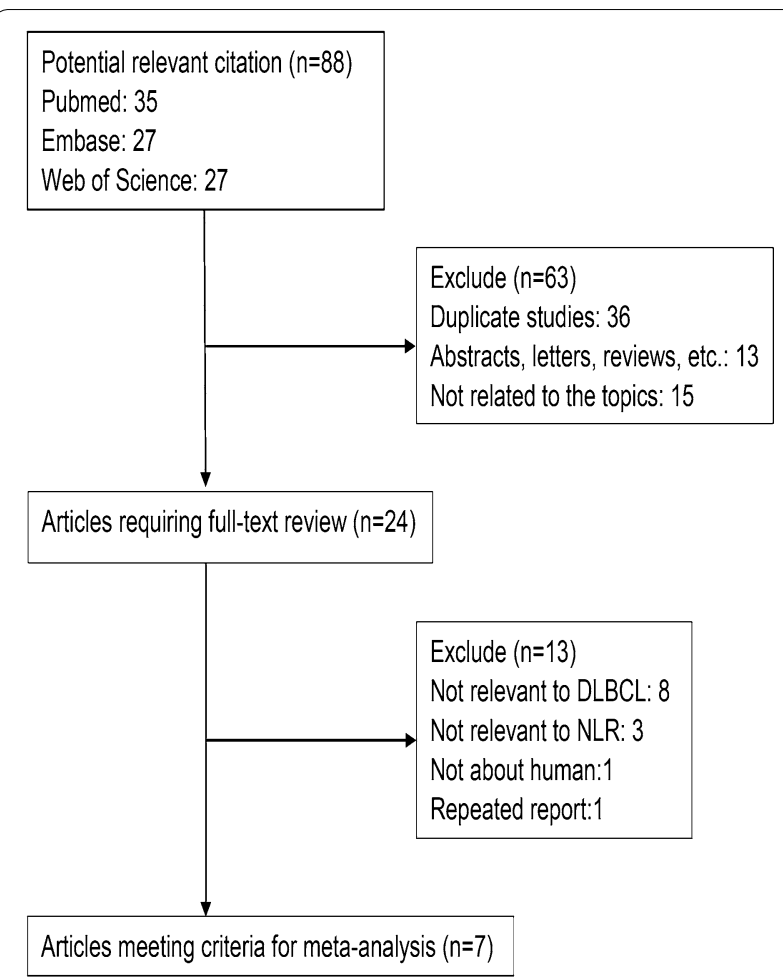

Fig. 1 Flow diagram of selecting relevant published works regarding NLR in DLBCL

with significant heterogeneity $\left(\chi^{2}=30.24, \quad p<0.001\right.$; $\mathrm{I}^{2}=76.9 \%$ ). Apparently, the study from Porrata et al. [28] might be the major source of the heterogeneity. After removing it from the enrolled studies, the pooled OR 2.477 (95\% CI 2.013-3.049) without any heterogeneity $\left(\mathrm{x}^{2}=4.09, p=0.664 ; \mathrm{I}^{2}=0 \%\right)$ indicated that higher NLR is significantly related to advanced Ann Arbor staging of DLBCL patients.

Moreover, we analyzed the association between NLR and other clinicopathological parameters, showing that higher NLR was significantly relevant to elder age, higher incidence rate of $\mathrm{B}$ symptoms and bone marrow involvement, poorer Eastern Cooperative Oncology Group-performance status (ECOG-PS), and higher lactate dehydrogenase (LDH) level (Table 2). However, the meta-analytic results presented us with no significant association between NLR and gender, extra nodal sites, and international prognostic index (IPI) score.

\section{Association between NLR and survival}

11 studies $[8,11,22-28]$ with 2454 patients in our analysis were included to examine the association between NLR and survival of DLBCL patients. With significant heterogeneity $\left(x^{2}=19.74, p=0.032 ; \mathrm{I}^{2}=49.3 \%\right)$, the pooled HR of 1.752 (95\%CI 1.464-2.096, Fig. 2) indicated 
Table 1 Characteristics of studies included in the meta-analysis

\begin{tabular}{|c|c|c|c|c|c|c|c|c|c|}
\hline Author & Year & Region & Follow-up (month) & Age (year) & No. (M/F) & Sample size & Outcome & Cut-off value & NOS score \\
\hline Porrata et al. [28] & 2010 & USA & $59.1(13.9-107.8)$ & $64(20-92)$ & $146 / 109$ & 255 & OS, PFS & 3.5 & 8 \\
\hline Ho et al. [11] & 2015 & China & 53.28 & $61.0(16-88)$ & $80 / 68$ & 148 & OS, PFS & 2.11 & 7 \\
\hline Keam et al. [23] & 2015 & Korea & $59.0(2.5-118.5)$ & $61(16-87)$ & $226 / 221$ & 447 & OS, PFS & 3 & 8 \\
\hline Melchardt et al. [9] & 2015 & Austria & 53 & $65.3(20-92)$ & $270 / 245$ & 515 & OS & 5.54 & 8 \\
\hline Ming et al. [29] & 2015 & China & NR & $55(20-85)$ & $24 / 27$ & 51 & OS & 2.32 & 6 \\
\hline Hong et al. [22] & 2016 & Korea & $48.9(46.5-51.3)$ & $56(16-86)$ & $177 / 136$ & 313 & PFS & 2.42 & 8 \\
\hline Ni et al. [27] & 2016 & China & NR & $54(14-75)$ & $36 / 23$ & 59 & OS, PFS & 2.915 & 6 \\
\hline Periša et al. [25] & 2016 & Croatia & $27(1-105)$ & $63(22-87)$ & $51 / 66$ & 117 & OS, EFS & 2.63 & 6 \\
\hline Wang et al. [26] & 2016 & China & $29(2-122)$ & NR & $90 / 66$ & 156 & OS, PFS & 3.0 & 6 \\
\hline Park et al. [24] & 2016 & Korea & NR & $60(32-81)$ & $53 / 46$ & 99 & OS, PFS & 3.5 & 6 \\
\hline Wang et al. [8] & 2017 & China & NR & $54(18-86)$ & $202 / 153$ & 355 & OS, PFS & 2.81 & 7 \\
\hline
\end{tabular}

NR not reported, OS overall survival, PFS progression free survival, EFS event free survival, NOS Newcastle-Ottawa Quality Assessment Scale

Table 2 Meta-analysis of the association between elevated NLR and clinicopathological parameters

\begin{tabular}{|c|c|c|c|c|c|}
\hline \multirow[t]{2}{*}{ Association } & \multirow[t]{2}{*}{ No. of studies } & \multirow[t]{2}{*}{ No. of patients } & \multirow[t]{2}{*}{ Pooled HR (95\% Cl) } & \multicolumn{2}{|c|}{ Heterogeneity } \\
\hline & & & & $\mathrm{I}^{2}(\%)$ & $p$-value \\
\hline Ann Arbor stage & 8 & 1525 & $2.121(1.367-3.293)$ & 76.9 & $<0.001$ \\
\hline$|\mathrm{P}|$ & 7 & 1170 & $1.869(0.881-3.962)$ & 88.8 & $<0.001$ \\
\hline B symptoms & 7 & 1474 & $1.867(1.034-3.372)$ & 84.3 & $<0.001$ \\
\hline BM involvement & 4 & 1004 & $1.550(1.148-2.094)$ & 8.0 & 0.353 \\
\hline ECOG-PS & 8 & 1525 & $2.786(2.067-3.755)$ & 0 & 0.673 \\
\hline Extranodal sites & 7 & 1422 & 1.314 (0.809-2.133) & 76.2 & $<0.001$ \\
\hline $\mathrm{LDH}$ & 8 & 1525 & $2.911(1.550-5.467)$ & 88.7 & $<0.001$ \\
\hline
\end{tabular}

IPI international prognostic index, BM bone marrow, ECOG-PS Cooperative Oncology Group performance status, $L D H$ lactate dehydrogenase

that patients with elevated NLR were expected to have shorter OS. Using restricted cubic spline function, we found non-linear associations between higher NLR and shorter OS in DLBCL patients $\left(\mathrm{P}_{\text {non-linearity }}=0.007\right.$; $\mathrm{chi}_{\text {model }}^{2}=26.88$ ).

Furthermore, 9 studies [8, 11, 22-25, 27, 28] were pooled to estimate the association between NLR and PFS/EFS in DLBCL patients. The analytic results showed a significant correlation between high NLR and shorter PFS/EFS. (HR 2.495, 95\% CI 2.208-2.819) without significant heterogeneity $\left(X^{2}=15.36, p=0.053\right.$; $\mathrm{I}^{2}=47.9 \%$ ) (Fig. 3). the dose-response meta-analysis showed a linear association between higher NLR and shorter OS in DLBCL patients treated with $\mathrm{R}-\mathrm{CHOP}$ $\left(\mathrm{P}_{\text {non-linearity }}=0.0011 ; \mathrm{chi}_{\text {model }}^{2}=24.12\right)$.

As we all know, OS and PFS/EFS are correlated with each other, and split-and-merge results will increase the probability of type I error. Therefore, the 3-level randomeffects meta-analysis model is applied using $\mathrm{R}$ software 3.2.2 [30] and meta SEM packages [31], verifying the overall effects of NLR on OS and PFS/EFS in DLBCL patients (HR 1.729, 95\% CI 1.221-2.448).

In addition, we combined HRs through cox multivariate analysis, to explore the independent prognostic role of NLR for OS and PFS/EFS in DLBCL patients although there was significant heterogeneity across the studies, pooled HRs and corresponding 95\% CIs of OS and PFS/ EFS were 1.752 (1.464-2.096) and 1.739 (1.255-2.411), respectively.

To explore the source of heterogeneity, subgroup analysis and meta regression were performed by the study location (eastern vs. western region), sample size $(\geq 200$ vs. $<200)$, cut-off value defining "elevated NLR" $(\geq 3$ vs. $<3)$, and NOS score $(\geq 7$ vs. $<7)$. The subgroup analysis did not alter the prognostic role of NLR in OS substantially (Table 3), with significant heterogeneity across studies in most subgroups. However, subgroup analysis of PFS/EFS by the study location showed obvious decreased heterogeneity. And meta-regression analysis revealed that the study location $(p 0.02)$ might explain 


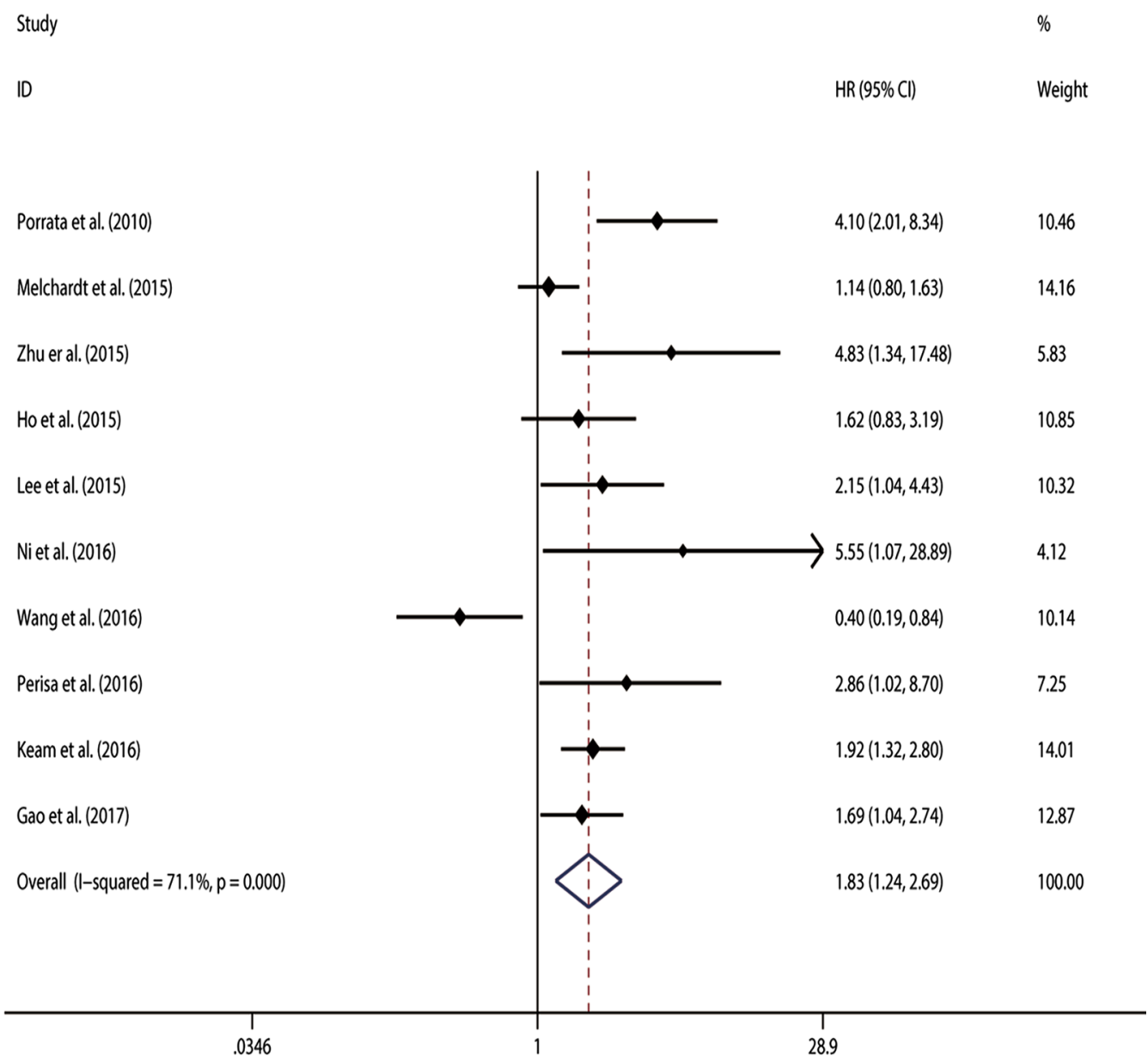

Fig. 2 Meta-analysis of the association between elevated NLR and OS in DLBCL

the source of the heterogeneity across the studies on PFS/ EFS (Table 4).

\section{Sensitivity analyses}

Sensitivity analyses were performed next. A single study involved in the meta-analysis was deleted each time to unveil the influence of the individual data set to the pooled HRs. In the current study, removing any of the included studies had no significant impact on the metaanalytic results, indicating the robustness of the results (Additional file 1: Figure S1).

\section{Publication bias}

The Begg's funnel plot showed no significant asymmetry for all included studies (Additional file 2: Figure S2). Accordingly, the $p$ value of Egger's test indicated that there was no publication bias among the studies included in our analysis $(p=0.792$ for OS, and $p=0.792$ for PFS/ EFS).

\section{Discussion}

Recently, several studies have shed light on the association between NLR and prognosis of cancer patients. However, these results are not comparable because of the heterogeneity on design and population, and the diversity in cut-off values defining "elevated NLR". A previous meta-analysis combining 9 studies has shown that NLR was a significant indicator for poor OS and PFS from a total of 2297 individuals [32]. Our study is an updated meta-analysis covering a total of 11 published studies with 2515 patients reporting the independent prognostic role of NLR in DLBCL patients treated with R-CHOP. Moreover, we conducted a dose-response meta-analysis to assess the association between NLR and risk of mortality from DLBCL among different cut-off values in the included studies. Because OS and PFS/EFS are correlated with each other, the 3-level random-effect meta-analysis model is applied to assess the prognostic role of NLR in DLBCL patients. 


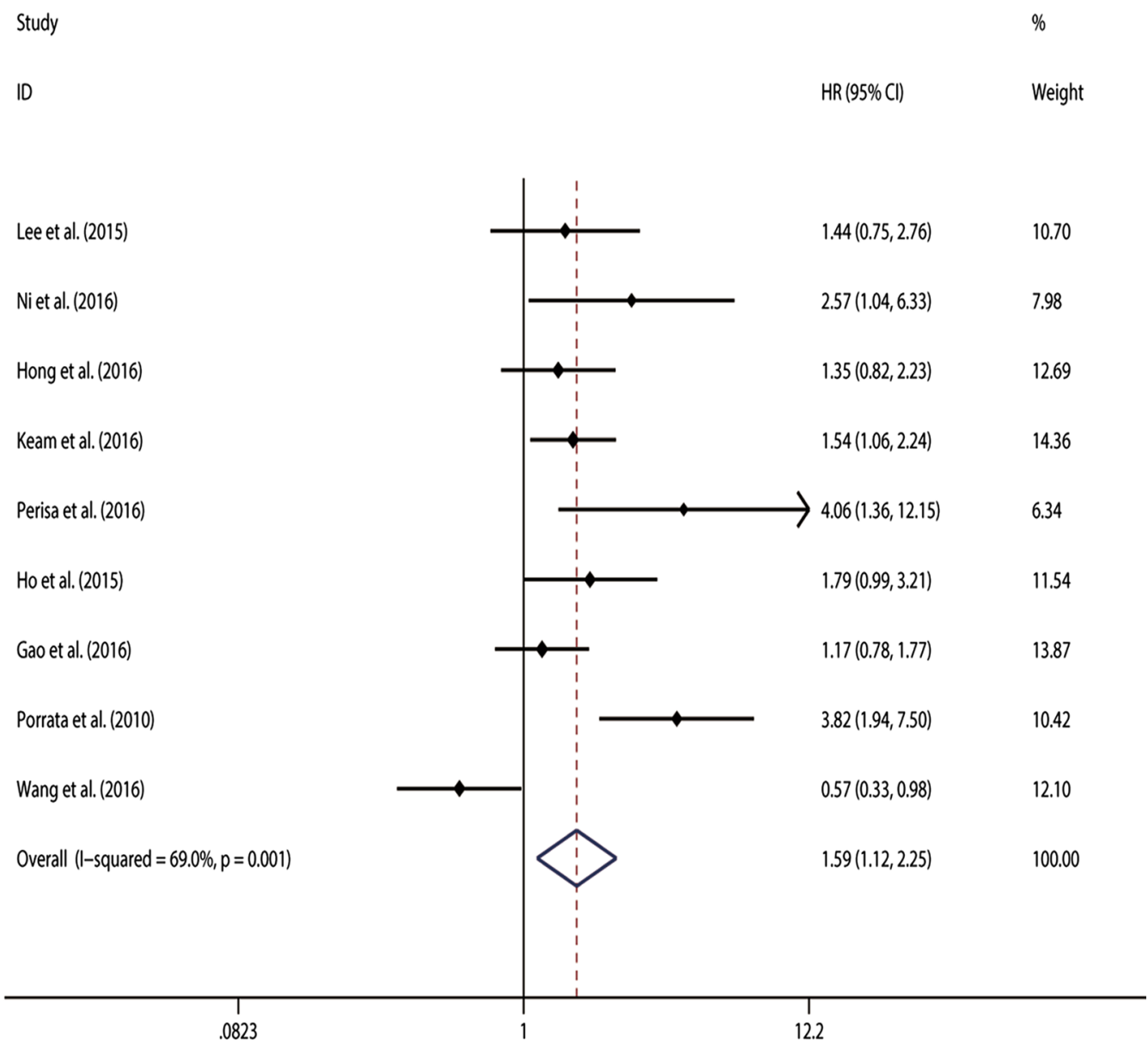

Fig. 3 Meta-analysis of the association between elevated NLR and PFS in DLBCL

Table 3 Subgroup analysis and Meta regression of pooled HRs for OS in DLBCL patients with high NLR

\begin{tabular}{|c|c|c|c|c|c|c|c|}
\hline \multirow[t]{2}{*}{ Subgroup analysis } & \multirow{2}{*}{$\begin{array}{l}\text { No. } \\
\text { of studies }\end{array}$} & \multirow[t]{2}{*}{ No. of patients } & \multicolumn{2}{|l|}{ Pooled HR (95\% Cl) } & \multirow{2}{*}{$\begin{array}{l}\text { Meta } \\
\text { regression } \\
\text { ( } p \text {-value) }\end{array}$} & \multicolumn{2}{|c|}{ Heterogeneity } \\
\hline & & & Fixed & Random & & $I^{2}(\%)$ & $p$-value \\
\hline \multicolumn{8}{|l|}{ Region } \\
\hline Western & 3 & 840 & $1.563[1.149-2.126]$ & $2.246[0.888-5.681]$ & & 82.2 & 0.004 \\
\hline Eastern & 8 & 1315 & 1.665 [1.317-2.105] & $1.701[1.050-2.756]$ & 0.657 & 69.8 & 0.003 \\
\hline \multicolumn{8}{|l|}{ Sample size } \\
\hline$<200$ & 6 & 616 & $1.542[1.077-2.209]$ & 1.921 [0.894-4.127] & 0.952 & 75.1 & 0.001 \\
\hline$\geq 200$ & 4 & 1539 & $1.659[1.334-2.064]$ & 1.841 [1.187-2.853] & & 72.6 & 0.012 \\
\hline \multicolumn{8}{|l|}{ Cut-off value } \\
\hline$<3.0$ & 5 & 823 & $2.539[1.784-3.612]$ & 2.908 [1.755-4.819] & 0.060 & 36.8 & 0.176 \\
\hline$\geq 3.0$ & 5 & 1332 & 1.368 [1.098-1.705] & $1.287[0.795-2.084]$ & & 75.5 & 0.003 \\
\hline \multicolumn{8}{|l|}{ NOS score } \\
\hline$\geq 7$ & 5 & 1687 & $1.656[1.345-2.038]$ & $1.787[1.242-2.571]$ & & 63.5 & 0.027 \\
\hline$<7$ & 5 & 468 & $1.627[1.350-1.960]$ & $1.826[1.238-2.692]$ & 0.949 & 80.1 & 0.000 \\
\hline
\end{tabular}

NOS Newcastle-Ottawa Quality Assessment Scale 
Table 4 Subgroup analysis and Meta regression of pooled HRs for PFS in DLBCL patients with high NLR

\begin{tabular}{|c|c|c|c|c|c|c|c|}
\hline \multirow[t]{2}{*}{ Subgroup analysis } & \multirow{2}{*}{$\begin{array}{l}\text { No. } \\
\text { of studies }\end{array}$} & \multirow[t]{2}{*}{ No. of patients } & \multicolumn{2}{|l|}{ Pooled HR (95\% CI) } & \multirow{2}{*}{$\begin{array}{l}\text { Meta regression } \\
(p \text {-value) }\end{array}$} & \multicolumn{2}{|c|}{ Heterogeneity } \\
\hline & & & Fixed & Random & & $I^{2}(\%)$ & $p$-value \\
\hline \multicolumn{8}{|l|}{ Region } \\
\hline Western & 2 & 358 & 3.885 [2.185-6.907] & 3.885 [2.185-6.907] & 0.027 & 0 & 0.926 \\
\hline Eastern & 7 & 1577 & $1.299[1.071-1.576]$ & 1.313 [0.973-1.772] & & 54.8 & 0.039 \\
\hline \multicolumn{8}{|l|}{ Sample size } \\
\hline$<200$ & 5 & 565 & 1.320 [0.972-1.792] & 1.588 [0.832-3.032] & 0.850 & 75.6 & 0.003 \\
\hline$\geq 200$ & 4 & 1370 & $1.530[1.209-1.742]$ & 1.641 [1.092-2.465] & & 66.1 & 0.031 \\
\hline \multicolumn{8}{|l|}{ Cut-off value } \\
\hline$<3.0$ & 4 & 830 & $1.445[1.209-1.742]$ & 1.660 [1.048-2.629] & 0.697 & 50.2 & 0.110 \\
\hline$\geq 3.0$ & 5 & 1105 & 1.449 [1.144-1.834] & $1.511[0.874-2.612]$ & & 79.8 & 0.001 \\
\hline \multicolumn{8}{|l|}{ NOS score } \\
\hline$\geq 7$ & 5 & 1518 & $1.561[1.262-1.931]$ & 1.648 [1.183-2.297] & & 56.0 & 0.059 \\
\hline$<7$ & 4 & 417 & $1.179[0.824-1.688]$ & $1.576[0.674-3.688]$ & 0.757 & 80.0 & 0.002 \\
\hline
\end{tabular}

NOS Newcastle-Ottawa Quality Assessment Scale

Several recent studies have demonstrated that the inflammatory nature of a tumor's microenvironment is a critical component of tumor initiation, growth, and progression $[33,34]$. Furthermore, a variety of systemic inflammatory biomarkers have been identified and studied in patients with DLBCL, such as NLR [35], serum LDH [36], serum C-reactive protein (CRP) [37], serum albumin [38], etc. NLR has the advantage of low economic cost and wide availability, thereby drawing increasing attention. Mechanically, an elevated NLR is usually caused by neutrophilia and lymphopenia. Neutrophilia may lead to secretion of vascular endothelial growth factor (VEGF) and suppression of cytolytic activity of immune cells such as lymphocytes, natural killer cells, and activated T cells, thus accelerating tumor progression $[39,40]$. Lymphopenia indicates disease severity and is linked to the immune escape of tumor cells from tumor-infiltrating lymphocytes (TILs) [41, 42]. Therefore, an elevated NLR generates a favorable immune microenvironment, thereby correlating to poor prognosis of patients.

The meta-analysis had some limitations that called for cautious interpretation of the results. First, only 11 studies published in full-text were included in this meta-analysis. Some information was missed and individual patient data were unattainable, which might decrease the accuracy of the results. Second, the cutoff value defining high NLR varied among individual studies (Table 1), which may have contributed to heterogeneity. Third, differences of paper quality and sample size across the studies might cause bias in the meta-analysis although subgroup analysis and meta regression did not show the paper quality or sample size as the resource of heterogeneity. Forth, most of the included studies reported positive results, therefore our results might overestimate the prognostic significance of NLR to some degree. However, there was no significant publication bias of the enrolled studies on OS or PFS/EFS.

Despite the above limitations, our meta-analysis supports the values of NLR as a promising independent risk factor of survival in DLBCL patients. NLR can be easily obtained from routine blood tests, thus intermediate assessments about changes in NLR during therapy were simply available. Keam et al. revealed that a reduction in NLR after R-CHOP therapy in patients with high pre-NLR was associated with better prognosis. Therefore, combining NLR with the IPI score helps to identify higher risk patients who need additional consolidation therapy, such as autologous stem cell transplantation. That is, NLR can help personalize the treatment intensity, as well as aftercare schedule, in order to increase the likelihood of early detection.

\section{Conclusion}

Here, we searched electronic databases for relevant studies, and enrolled 11 studies with a total of 2515 patients for meta-analysis, drawing a conclusion that patients with higher NLR were more likely to have poorer prognosis than those with lower NLR. Taken together, the results from our meta-analysis suggest that NLR gains a prognostic value for patients with DLBCL. More multi-center prospective cohorts are warranted to further validate the role of the NLR in DLBCL. 


\section{Additional files}

Additional file 1: Figure S1. Funnel plot of publication bias for (A) OS and (B) PFS.

Additional file 2: Figure S2. Sensitivity analysis of studies concerning NLR and (A) OS and (B) PFS

\section{Abbreviations}

NLR: neutrophil-lymphocyte ratio; DLBCL: diffuse large B-cell lymphoma; OR: odds ratio; HR: hazards ratio; Cl: confidence interval; LDH: lactate dehydrogenase; OS: overall survival; PFS: progression-free survival; EFS: event-free survival; NHL: non-Hodgkin's lymphomas; CHOP: standard cyclophosphamide, doxorubicin, vincristine, and prednisone chemotherapy: R-CHOP: rituximab immunotherapy to standard CHOP chemotherapy; ECOG-PS: Eastern Cooperative Oncology Group-performance status; NOS: the Newcastle-Ottawa Quality Assessment Scale; VEGF: vascular endothelial growth factor; TIL: tumorinfiltrating lymphocyte; IPI: international prognostic index.

\section{Authors' contributions}

SM and LA collected and analyzed the data, wrote the paper; FF and YQ extracted and analyzed the data; CS and $\mathrm{YH}$ conceived and designed this study, analyzed the data, wrote the paper; and all authors reviewed the paper. All authors read and approved the final manuscript.

\section{Author details}

${ }^{1}$ Institute of Hematology, Union Hospital, Tongji Medical College, Huazhong University of Science and Technology, Wuhan 430022, China. ${ }^{2}$ Cancer Center, Union Hospital, Tongji Medical College, Huazhong University of Science and Technology, Wuhan 430022, China.

\section{Acknowledgments}

We would like to thank the researchers and study participants for their contributions.

\section{Competing interests}

The authors declare that they have no competing interests.

\section{Availability of data and materials}

Please contact author for data requests.

\section{Consent for publication}

Not applicable.

\section{Ethics approval and consent to participate}

All procedures followed were in accordance with the ethical standards of the responsible committee on human experimentation (Research Ethics Committees of Wuhan Union Hospital, Tongji Medical College, Huazhong University of Science and Technology) and with the Helsinki Declaration of 1964 and later versions.

\section{Funding}

This work was supported by the Natural Science Funds of China (No. 81500172, No. 81202962, and No.81670197); and the Integrated Innovative Team for Major Human Diseases Program of Tongji Medical College, HUST; and the Clinical Research Physician Program of Tongji Medical College, HUST.

\section{Publisher's Note}

Springer Nature remains neutral with regard to jurisdictional claims in published maps and institutional affiliations.

Received: 20 March 2018 Accepted: 3 August 2018

Published online: 22 August 2018
References

1. Flowers CR, Sinha R, Vose JM. Improving outcomes for patients with diffuse large B-cell lymphoma. CA Cancer J Clin. 2010;60(6):393-408.

2. Friedberg JW. New strategies in diffuse large B-cell lymphoma: translating findings from gene expression analyses into clinical practice. Clin Cancer Res. 2011;17(19):6112-7.

3. Friedberg JW. Relapsed/refractory diffuse large B-cell lymphoma. Hematology. 2011;2011(1):498-505.

4. Vaidya R, Witzig TE. Prognostic factors for diffuse large B-cell lymphoma in the R (X) CHOP era. Ann Oncol. 2014;25(11):2124-33.

5. Rosenwald A, Wright G, Chan WC, Connors JM, Campo E, Fisher RI, Gascoyne RD, Mullerhermelink HK, Smeland EB, Giltnane JM. The use of molecular profiling to predict survival after chemotherapy for diffuse large-B-cell lymphoma. N Engl J Med. 2002;346(25):1937-47.

6. Hans CP, Weisenburger DD, Greiner TC, Gascoyne RD, Delabie J, Ott G, Müller-Hermelink HK, Campo E, Braziel RM, Jaffe ES. Confirmation of the molecular classification of diffuse large B-cell lymphoma by immunohistochemistry using a tissue microarray. Blood. 2004;103(1):275-82.

7. Mikhaeel NG. Interim fluorodeoxyglucose positron emission tomography for early response assessment in diffuse large B cell lymphoma: where are we now? Leuk Lymphoma. 2009;50(12):1931-6.

8. Wang J, Gao K, Lei W, Dong L, Xuan Q, Feng M, Wang J, Ye X, Jin T, Zhang $Z$, et al. Lymphocyte-to-monocyte ratio is associated with prognosis of diffuse large B-cell lymphoma: correlation with CD163 positive M2 type tumor-associated macrophages, not PD-1 positive tumor-infiltrating lymphocytes. Oncotarget. 2017;8(3):5414-25.

9. Melchardt T, Troppan K, Weiss L, Hufnagl C, Neureiter D, Trankenschuh W, Schlick K, Huemer F, Deutsch A, Neumeister P, et al. Independent prognostic value of serum markers in diffuse large B-Cell lymphoma in the era of the NCCN-IPI. J Natl Compr Cancer Netw (JNCCN). 2015;13(12):1501-8.

10. Gu XB, Tian T, Tian XJ, Zhang XJ. Prognostic significance of neutrophil-tolymphocyte ratio in non-small cell lung cancer: a meta-analysis. Sci Rep. 2015:5:12493.

11. Ho CL, Lu CS, Chen JH, Chen YG, Huang TC, Wu YY. Neutrophil/lymphocyte ratio, lymphocyte/monocyte ratio, and absolute lymphocyte count/absolute monocyte count prognostic score in diffuse large B-Cell lymphoma: useful prognostic tools in the rituximab era. Medicine. 2015:94(24):e993.

12. Templeton AJ, McNamara MG, Seruga B, Vera-Badillo FE, Aneja P, Ocana A, Leibowitz-Amit R, Sonpavde G, Knox JJ, Tran B, et al. Prognostic role of neutrophil-to-lymphocyte ratio in solid tumors: a systematic review and meta-analysis. J Natl Cancer Inst. 2014;106(6):dju124.

13. Viers BR, Boorjian SA, Frank I, Tarrell RF, Thapa P, Karnes RJ, Thompson RH, Tollefson MK. Pretreatment neutrophil-to-lymphocyte ratio is associated with advanced pathologic tumor stage and increased cancer-specific mortality among patients with urothelial carcinoma of the bladder undergoing radical cystectomy. Eur Urol. 2014;66(6):1157.

14. Altman DG, McShane LM, Sauerbrei W, Taube SE. Reporting Recommendations for Tumor Marker Prognostic Studies (REMARK): explanation and elaboration. PLoS Med. 2012;9(5):e1001216.

15. McShane LM, Altman DG, Sauerbrei W, Taube SE, Gion M, Clark GM REporting recommendations for tumor MARKer prognostic studies (REMARK). Nat Clin Pract Oncol. 2005;2(8):416-22.

16. Campo E, Swerdlow SH, Harris NL, Pileri S, Stein H, Jaffe ES. The 2008 WHO classification of lymphoid neoplasms and beyond: evolving concepts and practical applications. Blood. 2011;117(19):5019-32.

17. Parmar MK, Torri V, Stewart L. Extracting summary statistics to perform meta-analyses of the published literature for survival endpoints. Stat Med. 1998;17(24):2815-34.

18. Chuling F, Hui H, Zuojun X. The Newcastle-Ottawa Scale (NOS) for assessing the quality of nonrandomized studies. PLOS One. 2016. https://doi. org/10.1371/journal.pone.0154716.t002.

19. Orsini N, Bellocco R, Greenland S. Generalized least squares for trend estimation of summarized dose-response data. Stata J. 2006;6(6):40-57.

20. Desquilbet $L$, Mariotti F. Dose-response analyses using restricted cubic spline functions in public health research. Stat Med. 2010;29(9):1037-57.

21. Stuck AE, Rubenstein LZ, Wieland D, Vandenbroucke JP, Irwig L, Macaskill P, Berry G, Glasziou P, Seagroatt V, Stratton I. Bias in meta-analysis detected by a simple graphical test. BMJ. 1997;315(7129):629-34.

22. Hong JY, Ryu KJ, Lee JY, Park C, Ko YH, Kim WS, Kim SJ. Serum level of CXCL10 is associated with inflammatory prognostic biomarkers 
in patients with diffuse large B-cell lymphoma. Hematol Oncol. 2016;35:480-6.

23. Keam B, Ha H, Kim TM, Jeon YK, Lee SH, Kim DW, Kim CW, Heo DS. Neutrophil to lymphocyte ratio improves prognostic prediction of international prognostic index for patients with diffuse large B-cell lymphoma treated with rituximab, cyclophosphamide, doxorubicin, vincristine and prednisone. Leuk Lymphoma. 2015;56(7):2032-8.

24. Park LC, Lee HS, Lee EM, Shin SH, Kim YS. prognostic significance of neutrophil lymphocyte ratio and platelet lymphocyte ratio in diffuse large B-cell lymphoma patients treated with R-CHOP. Kosin Med J. 2016;31(2):122.

25. Periša V, Knezović A, Zibar L, Sinčić-Petričević J, Mjeda D, Periša I, Aurer I. Comparison of the prognostic impact of neutrophil/lymphocyte ratio, platelet/lymphocyte ratio, and glasgow prognostic score in diffuse large B-cell lymphoma. Shiraz E-Med J. 2016;17(7-8).

26. Wang J, Zhou M, Xu JY, Yang YG, Zhang QG, Zhou RF, Chen B, Ouyang J. Prognostic role of pretreatment neutrophil-lymphocyte ratio in patients with diffuse large B-cell lymphoma treated with RCHOP. Medicine. 2016;95(38):e4893.

27. Ni J, Wang YQ, Zhang YP, Wu W, Zeng QS, Yang MZ, Xia RX. Value of neutrophil/lymphocyte ratio and platelet/lymphocyte ratio for prognostic evaluation of diffuse large B-cell lymphoma. Zhongguo shi yan xue ye xue za zhi. 2016;24(2):427-32.

28. Porrata LF, Ristow K, Habermann T, Inwards DJ, Micallef IN, Markovic SN. Predicting survival for diffuse large B-cell lymphoma patients using baseline neutrophil/lymphocyte ratio. Am J Hematol. 2010;85(11):896-9.

29. Ming Z, Yanli L, Zhitaa W, Qianshan T, Zhimin Z. Neutrophil to lymphocyte ratio and its relationship with the chemotherapy effect and the prognosis in patients with diffuse large B-cell lymphoma. J Leuk Lymphoma. 2015;24(8):460-3 [Chinese]

30. Team RDC: R Core Team. R: A language and environment for statistica computing 2014. Viena: R Foundation for Statistical Computing; 2008.

31. Cheung MWL. metaSEM: an R package for meta-analysis using structural equation modeling. Front Psychol. 2015;5:1521.

32. Wang J, Zhou X, Liu Y, Li Z, Li X. Prognostic significance of neutrophil-tolymphocyte ratio in diffuse large B-cell lymphoma: a meta-analysis. PLoS ONE. 2017;12(4):e0176008.
33. Candido J, Hagemann T. Cancer-related inflammation. J Clin Immunol. 2013;33(1):79-84.

34. Carbone A, Tripodo C, Carlo-Stella C, Santoro A, Gloghini A. The role of inflammation in lymphoma. Adv Exp Med Biol. 2014;816(816):315-33.

35. Troppan K, Deutsch A, Gerger A, Stojakovic T, Behamschmid C, Wenzl K, Neumeister $P$, Pichler $M$. The derived neutrophil to lymphocyte ratio is an independent prognostic factor in patients with diffuse large B-cell lymphoma. Br J Cancer. 2014;1 10(2):369.

36. Jung SH, Yang DH, Ahn JS, Kim YK, Kim HJ, Lee JJ. Serum lactate dehydrogenase with a systemic inflammation score is useful for predicting response and survival in patients with newly diagnosed diffuse large B-cell lymphoma. Acta Haematol. 2014;133(1):10-7.

37. Troppan KT, Schlick K, Deutsch A, Melchardt T, Egle A, Stojakovic T, Behamschmid C, Weiss L, Neureiter D, Wenzl K. C-reactive protein level is a prognostic indicator for survival and improves the predictive ability of the R-IPI score in diffuse large B-cell lymphoma patients. Br J Cancer. 2014;111(1):55-60.

38. Dalia S, Chavez J, Little B, Bello C, Fisher K, Lee JH, Chervenick P, Sokol L, Sotomayor E, Shah B. Serum albumin retains independent prognostic significance in diffuse large B-cell lymphoma in the post-rituximab era. Ann Hematol. 2014;93(8):1305-12.

39. El-Hag A, Clark RA. Immunosuppression by activated human neutrophils. Dependence on the myeloperoxidase system. J Immunol. 1987;139(7):2406-13.

40. Petrie HT, Klassen LW, Kay HD. Inhibition of human cytotoxic T lymphocyte activity in vitro by autologous peripheral blood granulocytes. J Immunol. 1985;134(1):230.

41. Porrata LF, Rsitow K, Inwards DJ, Ansell SM, Micallef IN, Johnston PB, Habermann TM, Witzig TE, Colgan JP, Nowakowski GS. Lymphopenia assessed during routine follow-up after immunochemotherapy (R-CHOP) is a risk factor for predicting relapse in patients with diffuse large B-cell lymphoma. Leukemia. 2010;24(7):1343-9.

42. Raycoquard I, Cropet C, Van GM, Sebban C, Le CA, Judson I, Tredan O, Verweij J, Biron P, Labidi I. Lymphopenia as a prognostic factor for overall survival in advanced carcinomas, sarcomas, and lymphomas. Cancer Res. 2009:69(13):5383-91.
Ready to submit your research? Choose BMC and benefit from:

- fast, convenient online submission

- thorough peer review by experienced researchers in your field

- rapid publication on acceptance

- support for research data, including large and complex data types

- gold Open Access which fosters wider collaboration and increased citations

- maximum visibility for your research: over $100 \mathrm{M}$ website views per year

At $\mathrm{BMC}$, research is always in progress.

Learn more biomedcentral.com/submissions 